Kirkwood R.N., Thacker A., Gooneratne A.D., Guedo B.L., Laarveld B., 1988b. The influence of exogenous growth hormone on ovulation rate in gilts. Can. J. Anim. Sci., 68, 1097-1103.

Prunier A., Martin C., Mounier A.M., Bonneau M., 1993a. Metabolic and endocrine changes associated with undernutrition in the peripubertal gilt. J. Anim. Sci., 71, 1887-1894.

Prunier A., Etienne M., Dourmad J.Y., 1993b. Feeding level, metabolic parameters and reproductive performance of primiparous sows. Livest. Prod. Sci., 37, 185-196.

Quesnel H., Prunier A., 1995a. L'ovulation après le tarissement des truies : mécanismes physiologiques et facteurs de variation. INRA Prod. Anim., 8, 165176.

Quesnel H., Prunier A., 1995b. Endocrine bases of lactational anœstrus in the sow. Reprod. Nutr. Dev., $35,395-414$.

Quesnel H., Pasquier A., Mounier A.M., Prunier A., 1998a. Influence of feed restriction during lactation on gonadotropic hormones and ovarian development in primiparous sows. J. Anim. Sci., 76, 856-863.

Quesnel H., Pasquier A., Mounier A.M., Louveau I., Prunier A., 1998b. Influence of feed restriction in primiparous lactating sows on body condition and metabolic parameters. Reprod. Nutr. Dev., 38 (sous presse).

Rohner-Jeanrenaud E., Jeanrenaud B., 1997. Central nervous system and body weight regulation. Ann. Endocrinol. (Paris), 58, 137-142.
Rojkittikhun T., Uvnäs-Moberg K., Einarsson S., 1993. Plasma oxytocin, prolactin, insulin and $\mathrm{LH}$ after $24 \mathrm{~h}$ of fasting and after refeeding in lactating sows. Acta Physiol. Scand., 148, 413-419.

Tokach M.D., Pettigrew J.E., Dial G.D., Wheaton J.E., Crooker B.A., Johnston L.J., 1992a. Characterization of luteinizing hormone secretion in the primiparous lactating sow : relationship to blood metabolites and return-to-estrus interval. J. Anim. Sci., 70, 2195-2201.

Tokach M.D., Pettigrew J.E., Dial G.D., Wheaton J.E., Crooker B.A., Koketsu Y., 1992b. Influence of glucose infusions on luteinizing hormone secretion in the energy-restricted primiparous lactating sow. J. Anim. Sci., 70, 2202-2206.

Varley M.A., Prime G.R., 1993. The effect of food intake on prolificacy and plasma progesterone concentrations in primiparous sows. Livest. Prod. Sci., 34, 267-279.

Zachov R.J., Magoffin D.A., 1997. Direct intraovarian effects of leptin : impairment of the synergistic action of insulin like growth factor-I on follicle-stimulating hormone-dependent estradiol- $17 \beta$ production by rat ovarian granulosa cells.

Zak L.J., Cosgrove J.R., Aherne F.X., Foxcroft G.R. 1997. Pattern of feed intake and associated metabolic and endocrine changes differentially affect postweaning fertility in primiparous lactating sows. J. Anim. Sci., 75, 208-216.

\section{Alimentation et gestion des réserves corporelles de la truie : conséquences sur sa longévité}

J.Y. DOURMAD, M. ÉTIENNE, J. NOBLET

INRA Station de Recherches Porcines, $\mathrm{Au}$ cours des dernières années, les performances de reproduction des truies se sont largement améliorées, pour atteindre aujourd'hui une moyenne de 23,2 porcelets sevrés par truie et par an, et près de 30 dans les meilleurs élevages. Cette amélioration s'explique en partie par l'accélération du rythme de reproduction, en particulier la réduction de la durée d'allaitement et de l'intervalle sevrage-saillie fécondante. La prolificité moyenne dans les élevages a également augmenté, après une période de relative stabilité, pour atteindre aujourd'hui 12 porcelets nés par portée, dont 11,3 nés vivants. Le développement récent des lignées hyperprolifiques accélère encore cette évolution, le nombre de porcelets par portée dans ces lignées étant proche de 14 dans les meilleurs élevages (près de 13 porcelets nés vivants). En lactation, si l'on considère à la fois les effets de l'accroissement de la taille de la portée allaitée et l'augmentation de la vitesse de croissance des porcelets, on peut estimer que le gain de poids moyen journalier de la portée, qui est un bon indicateur de la production laitière, s'est amélioré de plus de $50 \%$ au cours des 20 dernières années $(2500$ à $3000 \mathrm{~g} / \mathrm{j}$ vs 1500 à $2000 \mathrm{~g} / \mathrm{j})$.

Cette évolution des performances des truies en gestation et en lactation s'est accompagnée de profondes modifications dans leurs besoins nutritionnels, alors que leurs réserves adipeuses et leur capacité d'ingestion ont plutôt diminué. Dans les conditions optimales, une truie peut sevrer plus de 8 portées pendant sa carrière, soit plus de 100 porcelets au cours d'une vie productive d'environ 4 ans. Cependant, peu de femelles (environ $10 \%$ ) atteignent ce niveau de production et les truies sont réformées après 4,5 portées en moyenne, en ayant sevré moins de 45 porcelets. Ceci pénalise les performances économiques de l'élevage.

L'étude bibliographique de l'influence des apports alimentaires sur la longévité (Dourmad et al 1994) a montré qu'une bonne maî- 
Figure 1. Principaux facteurs de variation des réserves corporelles chez la truie.

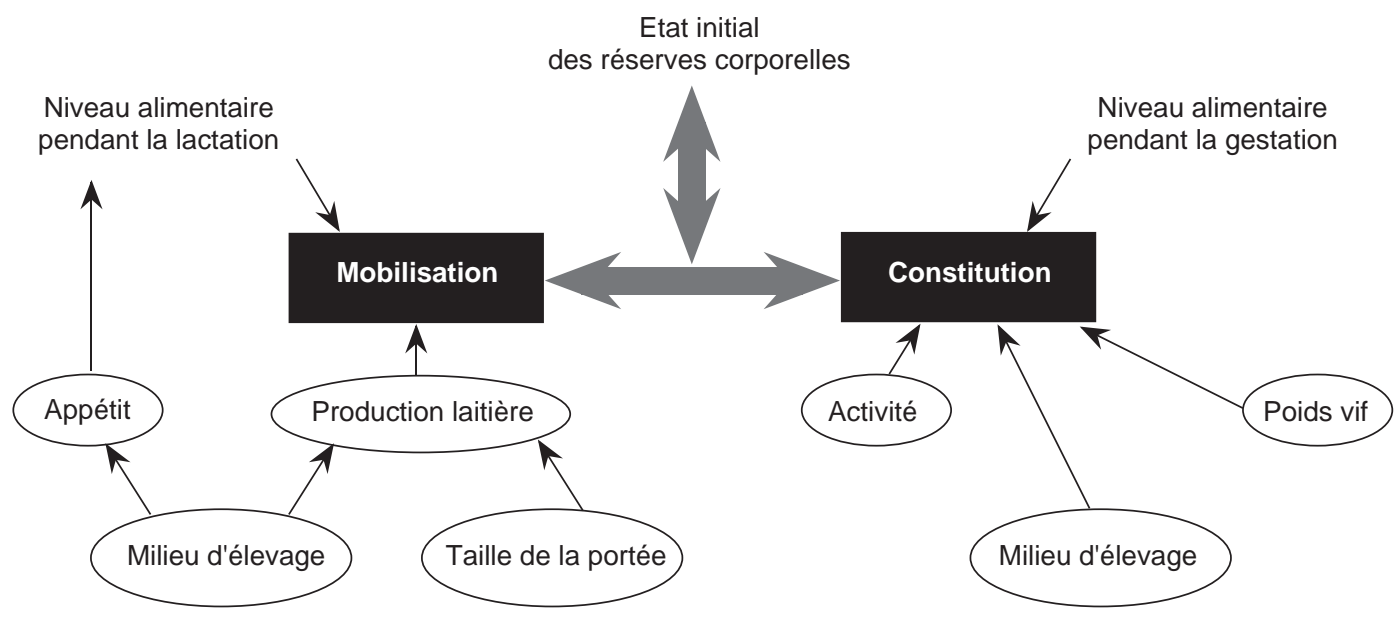

trise des apports de protéines et d'énergie est nécessaire pour optimiser la longévité de la truie. En effet, au cours des cycles successifs de reproduction, ses réserves corporelles fluctuent au rythme des gestations et des lactations qui se succèdent (figure 1). De nombreux travaux ont montré qu'une déplétion excessive des réserves corporelles pendant la lactation est préjudiciable aux performances ultérieures de reproduction. En fait, il semble exister un seuil critique en deçà duquel le retour en œstrus après le sevrage est retardé et le taux de fécondation diminué, en particulier après la première lactation. Les causes n'en sont pas encore élucidées, mais le statut métabolique et l'état des réserves adipeuses ou protéiques au moment du sevrage paraissent impliqués (Quesnel et Prunier 1995). A l'inverse, un poids vif élevé et un état d'engraissement excessif en fin de gestation accroissent les risques d'apparition de problèmes péri-partum et de réforme en raison de troubles de la locomotion. La plupart des effets de l'alimentation sur la reproduction semblent donc associés à des variations extrêmes des réserves corporelles (Dourmad et al 1994). Aussi, il est important de suivre une stratégie alimentaire adaptée à chaque truie, en relation avec son propre niveau de production, son comportement et le milieu d'élevage, de façon à maintenir pendant toute la carrière les réserves corporelles dans la zone optimale permettant de limiter les troubles de la reproduction et de maximiser la longévité. En pratique, l'estimation des réserves corporelles de la truie peut être réalisée à partir de la mesure de l'épaisseur de lard dorsal et du poids vif (Dourmad et al 1997), ou encore à l'aide d'un système de notation basé sur la description visuelle de certains repères anatomiques.

Les recommandations nutritionnelles moyennes, telles qu'elles étaient proposées jusqu'à présent (INRA 1989) sont peu adaptées pour prendre en compte cette nécessité d'individualiser les apports alimentaires, d'autant moins que l'on assiste à une certaine diversification des objectifs et des modes de production. Les conditions de production peuvent différer de façon importante entre les élevages, en relation avec le type de logement ou encore avec la saison. Au sein d'un même élevage, on note également une variabilité importante des besoins entre animaux, en fonction de l'âge, du numéro de portée et des performances. C'est pourquoi l'approche factorielle, qui consiste à quantifier les différentes dépenses des animaux et l'influence des facteurs de variation, remplace progressivement l'utilisation de recommandations moyennes. Elle permet de mieux adapter les apports à chaque situation d'élevage, voire à chaque animal. Cette approche a été largement utilisée pour déterminer les besoins énergétiques des truies en gestation et en lactation (Noblet et al 1990) et, dans une moindre mesure, les besoins en acides aminés (Dourmad et al 1991). Bien que très utile en pratique, l'approche factorielle présente cependant des limites. En effet, elle ne permet pas de prédire la réponse des animaux aux apports de nutriments, alors que la connaissance de cette réponse est souvent nécessaire pour définir une stratégie d'alimentation à long terme. C'est pourquoi des modèles plus dynamiques d'utilisation des nutriments par la truie sont à l'étude.

Dans l'état actuel des connaissances, il est donc possible de définir une stratégie alimentaire propre à chaque truie du troupeau, en fonction de ses performances antérieures et des objectifs de production. Des travaux à venir permettront sûrement d'améliorer la précision de cette prédiction, ce qui renforcera encore leur intérêt pratique. Néanmoins, ce niveau d'approche restera insuffisant pour intégrer les mécanismes physiologiques sousjacents, comme ceux qui sont impliqués dans les interactions nutrition - reproduction. Des modèles plus mécanistes, fonctionnant sur des pas de temps plus courts avec un niveau d'approche plus fin (organe ou cellule) seront nécessaires pour prendre en compte ces effets, mais leurs bases biologiques sont encore insuffisantes pour envisager de les utiliser en pratique. 


\section{Références}

Dourmad J.Y., Étienne M., Noblet J., 1991. Contribution à l'étude des besoins en acides aminés de la truie en lactation. Journées Rech. Porcine en France, 23, 61-68.

Dourmad J.Y., Étienne M., Prunier A., Noblet J., 1994. The effect of energy and protein intake of sows on their longevity : a review. Livest. Prod. Sci., 40, 87-97.

Dourmad J.Y., Étienne M., Noblet J., Causeur D., 1997. Prédiction de la composition chimique des truies reproductrices à partir du poids vif et de l'épaisseur de lard dorsal. Journées Rech. Porcine en France, 29, 255-262.

INRA, 1989. L'alimentation des animaux monogastriques. INRA Editions, Paris.

Noblet J., Dourmad J.Y., Étienne M., 1990. Energy utilization in pregnant and lactating sows : modeling of energy requirements. J. Anim. Sci., 68, 562-572.

Quesnel H., Prunier A., 1995. L'ovulation après le tarissement des truies : mécanismes physiologiques et facteurs de variation. INRA Prod. Anim., 8, 165176.

\section{Facteurs de variation de l'appétit des truies en lactation}

Au cours de ces dernières années, le nombre de porcelets produits et sevrés par truie s'est accru. Dans le même temps, l'appétit des truies en lactation n'a pas augmenté, voire a diminué, accentuant ainsi le déficit nutritionnel auquel la femelle est soumise pendant cette période d'exportation intense de nutriments. Ce phénomène pose le problème de l'élaboration d'une stratégie d'alimentation puisqu'il faut faire coïncider les besoins nutritionnels et la capacité des truies à ingérer suffisamment d'aliment. En fait, il apparaît que la consommation moyenne journalière $(\mathrm{CMJ})$ des truies est influencée à la fois par des facteurs intrinsèques de l'animal mais également par des facteurs extrinsèques, liés aux conditions d'élevage. L'étude de ces facteurs a déjà fait l'objet d'une revue par Dourmad (1988), ce texte l'actualise avec les résultats publiés depuis.

Figure 1. Effet marginal de la parité sur la consommation moyenne journalière d'aliment $(C M J)$ pendant la lactation (d'après O'Grady et al 1985).

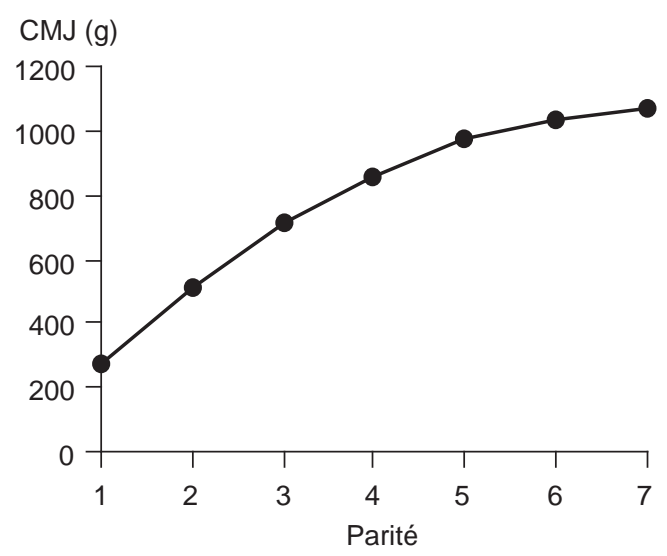

\section{1 / Caractéristiques de la truie}

\section{Numéro de portée}

L'augmentation de la CMJ avec la parité est principalement observée au cours des premières portées. En effet, les travaux de O'Grady et al (1985) mettent en évidence une augmentation curvilinéaire de la CMJ (figure 1) jusqu'à la $6^{\mathrm{e}}$ portée, alors qu'au-delà elle plafonne (Lynch 1989). Cependant, il s'agit de prendre en compte également l'augmentation parallèle de la taille de la portée et du poids de la truie, qui s'accompagnent d'un accroissement des besoins d'entretien et de production et donc potentiellement de la CMJ.

\section{Taille de la portée}

L'augmentation du nombre de porcelets s'accompagne d'un accroissement de la production quotidienne de lait (Elsley 1971) et, souvent, d'une mobilisation accrue des réserves corporelles. En effet, d'après Lynch (1989), la CMJ augmente en moyenne de $200 \mathrm{~g} / \mathrm{j}$ par porcelet alors que le besoin en aliment correspondant serait de 500 à $600 \mathrm{~g}$ (Verstegen et al 1985). L'effet du nombre de porcelets sur la CMJ est cependant de moins en moins important quand la taille de la portée s'accroît (O'Grady et al 1985).

\section{Stade de lactation}

La CMJ augmente avec le stade de lactation pour atteindre un plateau, mais, en pratique, les principales variations de CMJ pendant la lactation sont dues à la conduite alimentaire imposée au cours de la première semaine suivant la mise bas. A partir d'un grand nombre de données collectées sur le terrain, Koketsu et al (1996) ont pu établir six
N. QUINIOU, J.-Y. DOURMAD*, J. NOBLET*

Institut Technique du Porc, La Motte-au-Vicomte, 35650 Le Rheu

: $\square$ INRA, Station de Recherches

Porcines, 35590 Saint-Gilles 